\title{
Anterior subtemporal approach for posteriorly-projecting posterior communicating artery aneurysms
}

\author{
Tetsuyoshi Horiuchi, M.D., Fukuo Nakagawa, M.D., \\ Yuichiro Tanaka, M.D., Hiroshi Miyama, M.D., and Kazuhiro Hongo, M.D. \\ Department of Neurosurgery, \\ Azumino Red Cross Hospital, \\ Azumino, Japan \\ Department of Neurosurgery, \\ Shinshu University School of Medicine, \\ Matsumoto, Japan
}

\section{Correspondence to:}

Tetsuyoshi Horiuchi, M.D.

Department of Neurosurgery,

Shinshu University School of Medicine,

3-1-1 Asahi, Matsumoto 390-8621, Japan

Telephone: 81-263-37-2690

Fax: 81-263-37-0480

E-mail: thoriuchi-nsu@umin.ac.jp 


\begin{abstract}
Objective: We report our experience with the anterior subtemporal approach for the posterior communicating artery aneurysm protruding posteriorly.

Methods: Between 2000 and 2005, seven patients with posterior communicating artery aneurysm were operated on through the anterior subtemporal approach.

Results: The approach provided a better view than the pterional approach.

Conclusion: This approach seems to be suitable for posteriorly projecting posterior communicating artery aneurysms. The advantages of the anterior subtemporal approach are as follows: 1. It provides a short and a direct trajectory to the aneurysm. 2. Aneurysmal neck and surrounding structures can be easily identified and secured compared with the pterional approach. 3. A previously-placed clip for a middle cerebral artery or internal carotid artery aneurysm through the pterional route dose not interfere the clipping surgery for regrown or de novo posterior communicating artery aneurysms.
\end{abstract}

Running title: subtemporal approach for ICA aneurysm

Keywords: aneurysm, clipping, posterior communicating artery, surgery 


\section{Introduction}

The posterior communicating artery aneurysm (arising at the junction of internal carotid and posterior communicating arteries) is a common source of subarachnoid hemorrhage. The aneurysm usually projects postero-laterally and is well obliterated by the pterional approach [4,6,7]. However, in case of the aneurysm projecting posteriorly (or postero-medially), it is difficult to identify the posterior communicating artery and to confirm the patency of parent artery and its perforators after clip placement through the pterional approach. To overcome this problem, we use the anterior subtemporal approach for better visualization of an aneurysmal neck, posterior communicating artery and other structures.

In this report, we discuss the indications, surgical technique, and pitfalls to this approach.

\section{Clinical Material and Methods}

Patient Population: Between 2000 and 2005, seven patients with posterior communicating artery aneurysm, who underwent clipping surgery via the anterior subtemporal approach at the Department of Neurosurgery, Azumino Red Cross Hospital, were included in this study. Basically, the anterior subtemporal approach in stead of the pterional approach was chosen in patients with the aneurysm projecting posteriorly (or postero-medially) and the posterior communicating artery originating from the ventral surface of the internal carotid artery. Although the subtemporal approach was difficult to access the aneurysm if the aneurysmal neck located higher than usual, the subtemporal approach was not abandoned with the neck location in the present study.

Surgical Technique: A patient was placed supine on the table with a roll beneath the ipsilateral shoulder. The head was rotated until the temporal region lay horizontal with vertex down. A semicircular incision was made from just below the zygoma to the midline. The zygoma was exposed. The dissection was maintained deep to the temporal fascia to avoid injury the facial nerve. The zygomatic arch was temporaily removed and the temporalis muscle was retracted inferiorly. The fronto-temporal craniotomy was carried out (Figure 1). Using malleable brain retractors, the temporal lobe was gently elevated and the tentorial edge was visualized. The course of the internal carotid artery was identified and the arachnoid covering the internal carotid artery was meticulously cut to allow cerebrospinal fluid drainage and brain relaxation. Surgeons should be aware that the aneurysm located around the tentorial edge. Then, the proximal internal carotid artery was secured for the 
proximal control. Usually, a part of the posterior projecting aneurysm was located under the tentorial edge. To obtain sufficient operative filed, the incision of the tentorium was helpful (Figure 1). A straight or bayonet clip was placed not to injure the posterior communicating artery and its perforators. After the aneurysm was clipped, the dura mater was closed. The bone flap was affixed and the scalp was closed in layers.

\section{Illustrative cases}

Patient 2: A 48-year-old woman suddenly suffered a severe headache. Computed tomography showed a subarachnoid hemorrhage and cerebral angiography detected two internal carotid artery aneurysms on the right side (Figure 2). The ruptured posterior communicating artery aneurysm was clipped via the anterior subtemporal approach (Figure 3). However, the anterior choroidal artery aneurysm was intentionally left not to damage the temporal lobe due to excessive brain retraction. The postoperative course was uneventful and follow-up angiograms disclosed complete clipping (Figure 2) On Day 41, the unruptured anterior choroidal artery aneurysm was obliterated via the pterional approach (Figure 3). The patient was discharged without neurological deficits.

Patient 6: A 78-year-old woman suffered a subarachnoid hemorrhage caused by a ruptured right middle cerebral artery aneurysm in 1993, at the age of 68 years. The aneurysm was obliterated and no other aneurysms were observed at that time. In 1995, the patient complained of a headache and follow-up angiograms detected a de novo right posterior communicating artery aneurysm and the regrowth of the middle cerebral artery aneurysm (Figure 4). The second surgery was carried out in 1997. Aneurysms were clipped via the pterional route and postoperative angiograms showed complete clipping (Figure 4). In March 2003, the posterior communicating artery aneurysm re-grew and was partially embolized with coil (Figure 4). The right oculomotor nerve palsy developed in June 2003. Follow-up angiograms demonstrated a coil compaction and an enlargement of the residual aneurysm. Therefore, the aneurysm was obliterated through the anterior subtemporal approach (Figure 4 and 5). Postoperatively, the patient fully recovered.

\section{Results}

Table 1 shows clinical characteristics of seven patients in the present study. Successful and complete clipping was achieved in all patients via the anterior subtemporal 
approach. A ventricular drainage tube was placed to evacuate the cerebrospinal fluid in four patients. There was no surgery-related mortality. Two patients (Patient 5 and 7) developed oculomotor palsy after surgery and asymptomatic temporal contusion occurred in Patient 5.

\section{Discussion}

When compared with the pterional approach, the anterior subtemporal approach for posteriorly projecting posterior communicating artery aneurysms has three major advantages. The first is that it provides a short and direct trajectory. Minimum exposure of the aneurysm with less dissection allows surgeons to obtain sufficient operative field for clipping. The second advantage of the approach is that a surgeon can easily observe the aneurysmal neck and perforating arteries without surgical mirror, endoscope, and direct retraction of the internal carotid artery. The approach provided excellent visualization of the neck and adjacent neurovascular structures. The third advantage is that the approach is useful in case of de novo aneurysm or regrowth of the aneurysm. Because a previously-applied clip via pterional approach is often an obstacle at the second surgery.

The preoperative oculomotor nerve palsy developed in approximately $40 \%$ of patients having the posterior communicating artery aneurysm [6,7]. The palsy is usually recovered over weeks to months. Yasargil [7] reported there were no patients with permanent oculomotor nerve palsy associated with surgery via the pterional approach. In the present series, there were two patients (29\%; Patient 1 and 6) having the oculomotor nerve palsy preoperatively. Patient 6 fully recovered and Patient 1 died due to severe vasospasm. Two patients (Patient 5 and 7) suffered from the palsy after surgery. The palsy would be caused by the heat injury related to the bipolar coagulator or the sinus packing to obtain hemostasis of venous bleeding at the cut tentorial edge. The surgeon should be aware of the increased incidence of the oculomotor nerve injury via the anterior subtemporal approach rather than the pterional approach.

It would be difficult to know the relationship between the aneurysm and the oculomotor nerve preoperatively. Additionally, the course of the oculomotor nerve in the tentorium and cavernous sinus varies in individuals. Magnetic resonance cisternography would be useful to know the aneurysm and surrounding structures. Unfortunately, magnetic resonance cisternography was not performed in the present study.

The posterior communicating artery is a common aneurysm site encountered by neurosurgeons. By no means do we view our present approach, the aneurysm was usually 
obliterated through the pterional approach. The subtemporal approach was initially popularized by Drake [2] to treat basilar artery aneurysms. Subsequent modification of the anterior subtemporal approach has been used to reach the brainstem. There are some limitations of the subtemporal approach, including narrow operative field, temporal lobe contusion, clot removal, setting of cerebrospinal drainage, and, oculomotor nerve injury. In the present study, one patient who did not undergo cerebrospinal fluid drainage, developed asymptomatic temporal lobe contusion associated with a brain retractor. Therefore, placement of a spinal or ventricular drainage tube is recommended to avoid brain contusion. In our experience, the another disadvantage of the subtemporal approach , when compared with the pterional approach, has been the increase in operative time required for the opening and the closure.

The anterior temporal approach combined with the pterional approach is also used for basilar aneurysms $[1,3,5]$. The anterior temporal approach would be useful in patients with the posteriorly projecting posterior communicating artery aneurysm, because the aneurysmal complex can be observed from more lateral side. However, to retract the temporal lobe posteriorly, the veins from the temporal tip to the cavernous sinus and sphenoparietal sinuses are sacrificed in the anterior temporal approach. Therefore, there is a potential complication associated with venous injury. In the present cases, all veins were well preserved and there were no troubles associated with vessel injury. Additionally, we believe that the subtemporal approach has the significant advantage over the other approach in the visualization of the course of the posterior communicating artery and its perforators because these arteries can be observed from the more lateral side (see Figure 3).

\section{Conclusions}

The anterior subtemporal approach provides excellent visualization of a posteriorly projecting posterior communicating artery aneurysm projecting posteriorly. Since neurosurgeon can see the important structures through the subtemporal approach, one can perform the more complete obliteration of the aneurysm comparing with other approaches. 


\section{Reference List}

1. Bendok BR, Getch CC, Parkinson R, et al. (2004) Extended lateral transsylvian approach for basilar bifurcation aneurysms. Neurosurgery 55:174-178

2. Drake CG (1961) Bleeding aneurysms of the basilar artery. Direct surgical management in four cases. J.Neurosurg. 18:230-238

3. Heros RC, Lee SH (1993) The combined pterional/anterior temporal approach for aneurysms of the upper basilar complex: technical report. Neurosurgery 33:244-250

4. Lee KC, Lee KS, Shin YS, et al. (2003) Surgery for posterior communicating artery aneurysms. Surg.Neurol. 59:107-113

5. Sano K, Shiokawa Y (1994) The temporo-polar approach to basilar artery aneurysms with or without zygomatic arch translocation. Acta Neurochir.(Wien.) 130:14-19

6. Schmidek HH Surgical management of posterior communicating, anterior choroidal, and carotid bifurcation aneurysms, in Schmidek HH (ed): Schmidek \& Sweet Operative Neurosurgical Techniques: Indications, Methods, and Results. Vol 1. Philadelphia: WB Saunders, 2000, pp 1129-1134

7. Yasargil MG Internal carotid artery aneurysms, in Yasargil MG (ed): Microneurosurgery Vol 2. Stuttgart: Georg Thieme Verlag, 1984, pp 71-98 


\section{Figure legends}

Figure 1: A: Right frontotemporal craniotomy and zygomatic osteotomy. B: Exposure of the posterior communicating artery aneurysm via the anterior subtemporal approach.

Figure 2: Patient 2. Preoperative (upper column) right internal carotid angiograms disclosing the posterior communicating artery and anterior choroidal artery aneurysms. Postoperative angiograms (lower column) showing complete clipping of the posterior communicating artery aneurysm. A and C: anteroposterior view, B and D: lateral view.

Figure 3: Patient 2. A and B: Operative photographs in the first surgery via the anterior subtemporal approach showing the posterior communicating artery aneurysm before (A) and after (B) clipping. C: Operative photograph in the second surgery via the pterional approach demonstrating the bayonet clip and the anterior choroidal artery aneurysm. Note that the posterior communicating artery and its perforating arteries can be visualized via the anterior subtemporal approach, but not the pterional approach.

Figure 4. Patient 6. Right internal carotid angiograms (lateral view). A: Follow-up angiogram after the first direct surgery showing a de novo posterior communicating artery aneurysm and regrowth of the middle cerebral artery aneurysm. B: Postoperative angiogram after the second direct surgery disclosing complete obliterations of two aneurysms. C: Follow-up angiogram after the second direct surgery revealing regrowth of the posterior communicating artery aneurysm. D: Postoperative angiogram after the third direct surgery via the anterior subtemporal approach demonstrating complete clipping of the aneurysm.

Figure 5. Patient 6. Operative photographs via the anterior subtemporal approach showing the regrowth of the posterior communicating artery aneurysm before (A) and after (B) clipping. Arrowhead indicating the previously-applied straight clip. 
Table 1. Clinical Features

\begin{tabular}{|c|c|c|c|c|c|c|c|}
\hline Patient No. & $\begin{array}{l}\text { Age (yrs)/ } \\
\text { Sex }\end{array}$ & $\begin{array}{c}\text { Preoperative } \\
\text { WFNS } \\
\text { Grade }\end{array}$ & Side & $\begin{array}{l}\text { Size } \\
(\mathrm{mm})\end{array}$ & $\begin{array}{l}\text { Outcome } \\
\text { (GOS) }\end{array}$ & Complications & $\begin{array}{c}\text { Other } \\
\text { Characteristics }\end{array}$ \\
\hline 1 & $34 / \mathrm{M}$ & V & $\mathrm{R}$ & 5 & $\mathrm{D}$ & none & Ventricular drainage \\
\hline 2 & $48 / \mathrm{F}$ & I & $\mathrm{R}$ & 7 & GR & none & Ventricular drainage \\
\hline 3 & $62 / \mathrm{F}$ & I & $\mathrm{R}$ & 4 & GR & none & Ventricular drainage \\
\hline 4 & $67 / \mathrm{M}$ & 0 & $\mathrm{R}$ & 7 & $\mathrm{D}$ & Pulmonary embolism & Ventricular drainage \\
\hline 5 & $73 / F$ & III & $\mathrm{R}$ & 8 & MD & $\begin{array}{l}\text { Temporal lobe contusion } \\
\text { Mild oculomotor palsy }\end{array}$ & \\
\hline 6 & $78 / \mathrm{F}$ & 0 & $\mathrm{R}$ & 10 & GR & none & De novo aneurysm \\
\hline 7 & $84 / \mathrm{F}$ & II & $\mathrm{R}$ & 13 & SD & Severe oculomotor palsy & Left hemiparesis before ictus \\
\hline
\end{tabular}

WFNS: World Federation of Neurosurgical Societies, GOS: Glasgow outcome scale, F: female, M: male, R: right, GR: good recovery, MD: moderate disability, SD: severe disability, D: dead 